\title{
Navigating the Auditory Scene: An Expert Role for the Hippocampus
}

\author{
Sundeep Teki, ${ }^{1 \star}$ Sukhbinder Kumar, ${ }^{1,2 \star}$ Katharina von Kriegstein, ${ }^{3}$ Lauren Stewart, ${ }^{4}$ C. Rebecca Lyness, ${ }^{5}$ \\ Brian C. J. Moore, ${ }^{6}$ Brian Capleton, ${ }^{7}$ and Timothy D. Griffiths ${ }^{1,2}$ \\ ${ }^{1}$ Wellcome Trust Centre for Neuroimaging, University College London, London, WC1N 3BG, United Kingdom, ${ }^{2}$ Auditory Group, Institute of Neuroscience, \\ Newcastle University Medical School, Newcastle upon Tyne, NE2 4HH, United Kingdom, ${ }^{3}$ Max Planck Institute for Human Cognitive and Brain Sciences, \\ 04103 Leipzig, Germany, ${ }^{4}$ Department of Psychology, Goldsmiths, University of London, London, SE14 6NW, United Kingdom, ${ }^{5}$ Institute of Cognitive \\ Neuroscience, University College London, London, WC1N 3AR, United Kingdom, ${ }^{6}$ Department of Experimental Psychology, University of Cambridge, \\ Cambridge, CB2 3EB, United Kingdom, and 7Royal National College, Hereford, HR1 1EB, United Kingdom
}

Over a typical career piano tuners spend tens of thousands of hours exploring a specialized acoustic environment. Tuning requires accurate perception and adjustment of beats in two-note chords that serve as a navigational device to move between points in previously learned acoustic scenes. It is a two-stage process that depends on the following: first, selective listening to beats within frequency windows, and, second, the subsequent use of those beats to navigate through a complex soundscape. The neuroanatomical substrates underlying brain specialization for such fundamental organization of sound scenes are unknown. Here, we demonstrate that professional piano tuners are significantly better than controls matched for age and musical ability on a psychophysical task simulating active listening to beats within frequency windows that is based on amplitude modulation rate discrimination. Tuners show a categorical increase in gray matter volume in the right frontal operculum and right superior temporal lobe. Tuners also show a striking enhancement of gray matter volume in the anterior hippocampus, parahippocampal gyrus, and superior temporal gyrus, and an increase in white matter volume in the posterior hippocampus as a function of years of tuning experience. The relationship with gray matter volume is sensitive to years of tuning experience and starting age but not actual age or level of musicality. Our findings support a role for a core set of regions in the hippocampus and superior temporal cortex in skilled exploration of complex sound scenes in which precise sound "templates" are encoded and consolidated into memory over time in an experience-dependent manner.

\section{Introduction}

We assessed brain specialization in a previously unstudied group of expert listeners who spend large amounts of time exploring a complex acoustic environment that requires accurate perception and adjustment. The subjects, piano tuners, perform an active listening and adjustment task every time a piano is tuned where they explore an acoustic scene according to a specific route. Piano tuning is a highly sophisticated skill: a single tuning session might take up to $2 \mathrm{~h}$ and a professional tuner may typically tune up to 20 pianos a week, so the highest levels of expertise follow thousands of hours of experience over a period of 20 to 30 years (Capleton, 2007). Thus, this group provides a unique opportunity to inves-

\footnotetext{
Received Jan. 6, 2012; revised April 21, 2012; accepted May 19, 2012.

Author contributions: S.K., K.v.K., L.S., B.C.M., B.C., and T.D.G. designed research; S.T., S.K., K.v.K., and L.S. performed research; S.T., S.K., K.v.K., L.S., and C.R.L. analyzed data; S.T. and T.D.G. wrote the paper.

This work was funded by the Wellcome Trust, UK. We are grateful to the Radiology and IT staff at the Wellcome Trust Centre for Neuroimaging for their excellent technical support. We thank Simon Baumann, Adrian Rees, and Eleanor Maguire for comments on this manuscript and John Ashburner for help with the volumetric analysis.

*S.T. and S.K. contributed equally to this work.

This article is freely available online through the J Neurosci Open Choice option.

Correspondence should be addressed to Sundeep Teki, Wellcome Trust Centre for Neuroimaging, University College London, 12 Queen Square, London, WC1N 3BG, UK. E-mail: sundeep.teki.10@ucl.ac.uk.

DOI:10.1523/JNEUROSCI.0082-12.2012

Copyright $\odot 2012$ the authors $\quad 0270-6474 / 12 / 3212251-07 \$ 15.00 / 0$
}

tigate neuroanatomical bases of skilled organization of sound scenes.

Piano tuning requires the practitioner to perform successive navigation between tuned and untuned notes in which the beat rate between notes is used as a form of "signpost." Traditionally, tuning starts with tuning a single note to a standard tuning fork. A two-note chord is then played, comprising the tuned note and another note. The two notes contain multiple frequencies at the fundamental and higher harmonics, and certain harmonics in the two notes occur close to each other and produce beats fluctuations in the envelope of the combined harmonics at a rate equal to their frequency difference (Fig. 1A). The piano tuner is required to detect a particular beat rate corresponding to the interval between the two notes played, and match that to a specific value. A further interval is then tuned based on the most recently tuned note and another note, and this process is repeated iteratively (Fig. $2 A$ ). The beat rate and the frequency region in which the beat is to be detected vary for different intervals but are fixed for any given interval with beat rates that are typically less than $20 \mathrm{~Hz}$ (Table 1 ).

The acoustic processes underlying tuning include: (1) selective listening and detection of beats within particular frequency regions, and (2) adjustment of the beat rate and comparison with a predefined rate that is dependent on the interval being tuned (which is learned and remembered by trained tuners). Impor- 
Table 1. Beat rate relationships for different piano note pairs (Capleton, 2007)

\begin{tabular}{|c|c|c|c|c|c|c|c|c|c|c|c|}
\hline Major third (4 st) & Beats (Hz) & Perfect fourth (5 st) & Beats (Hz) & Perfect fifth (7 st) & Beats (Hz) & Major sixth (9 st) & Beats (Hz) & Minor third (3 st) & Beats (Hz) & Minor sixth (8st) & Beats $(\mathrm{Hz})$ \\
\hline $\mathrm{F}-\mathrm{A}$ & 6.9 & $\mathrm{~F}-\mathrm{Bb}$ & 0.8 & $\mathrm{~F}-\mathrm{C}$ & 0.6 & $F-D$ & 7.9 & $\mathrm{~F}-\mathrm{Ab}$ & 9.4 & $\mathrm{~F}-\mathrm{Db}$ & 11.0 \\
\hline F\#-A\# & 7.3 & F\#-B & 0.8 & F\#-C\# & 0.6 & F\#-D\# & 8.4 & F\#-A & 10.0 & F\#-D & 11.7 \\
\hline$G-B$ & 7.8 & $G-C$ & 0.9 & $G-D$ & 0.7 & $\mathrm{G}-\mathrm{E}$ & 8.9 & $\mathrm{G}-\mathrm{Bb}$ & 10.6 & G-Eb & 12.3 \\
\hline $\mathrm{A}-\mathrm{C} \#$ & 8.7 & $A-D$ & 1.0 & $A-E$ & 0.7 & A-F\# & 10.0 & $A-C$ & 11.9 & $A-F$ & 13.9 \\
\hline $\mathrm{Bb}-\mathrm{D} \#$ & 9.2 & A\#-D\# & 1.1 & $\mathrm{Bb}-\mathrm{F}$ & 0.8 & $\mathrm{Bb}-\mathrm{G}$ & 10.6 & $\mathrm{A \# -C \#}$ & 12.6 & A\#-F\# & 14.7 \\
\hline B-D\# & 9.8 & $B-E$ & 1.1 & B-F\# & 0.8 & & & $B-D$ & 13.3 & $B-G$ & 15.6 \\
\hline D-F\# & 11.7 & D-G & 1.3 & & & & & & & & \\
\hline$E b-G$ & 12.3 & & & & & & & & & & \\
\hline
\end{tabular}

We simulated the process of listening to beats in a frequency window through the use of a five-element harmonic complex where only the middle component is modulated at a rate relevant to the tuning procedure. Table 1 shows the range of beat rates for different note pairs for the scale area F3-G4 (difference in semitones indicated at the top) corresponding to the intervals used in tuning. The major thirds, perfect fourths, perfect fifths, and major sixths are the most useful for tuning, but the minor thirds and sixths that are also useful are included as well. In the experiment we used rates of $2 \mathrm{~Hz}$ and $16 \mathrm{~Hz}$ that are both within this range. st, Semitones.

tantly, the first stage of active listening and beat detection represents an essential entry-level skill following which the second skill of beat rate adjustment and navigation in pitch space via different tuning algorithms may develop further. Interestingly, neither musical expertise nor absolute pitch is required for proficient piano tuning (Capleton, 2007).

Here, we measured thresholds of tuners and controls on an amplitude modulation rate discrimination task that simulated beat detection and investigated structural brain changes in tuners that are related to their tuning experience. Based on the requirements of tuning, we predicted structural changes in the auditory cortex of all tuners that correspond to their specific expertise in a specialized type of active listening. We also predicted structural changes in hippocampus that evolve over several years of practice during which tuners repeatedly encode beat-rate templates into particular spectral and pitch contexts.

\section{Materials and Methods}

Participants. Participants included 19 tuners (all members of the Pianoforte Tuners' Association, UK, aged 25-78 years; mean age, 51.5 years; average tuning experience: $27.7 \pm 16.5$ years (range: $2-50$ years); 3 females; 6 left-handed) and 19 age-matched controls (aged 25-69 years; mean age, 49.7 years; 2 females; 5 left-handed) with normal hearing and no history of audiological or neurological disorders. All the tuners tuned pianos by the ear without the use of any electronic instruments. All provided informed written consent. Each participant completed a musical training questionnaire and was categorized into one of seven categories according to their level of musical training (see Notes). No blind participants were studied. The average level of musical training of the control participants was matched to the average musical training level of the piano tuners. The study was approved by an NHS Research Ethics Committee (ref. 07/Q0905/30).

Psychophysics. The tests simulated in a generic way an essential element of piano tuning: detecting changes in beats that are heard within a particular spectral window. Stimuli consisted of a $2 \mathrm{~s}$ long, five-element harmonic complex tone with components at 333.3, 666.6, 1000, 1333.3, and $1666.6 \mathrm{~Hz}$. The $1000 \mathrm{~Hz}$ component only was modulated to simulate beating of one element within a harmonic complex as occurs in piano tuning: sinusoidal amplitude modulation (AM) with 50\% modulation depth was applied at a rate of 2 or $16 \mathrm{~Hz}$ with roving of the modulation rate by $\pm 10 \%$ between trials.

A two-interval two-alternative forced-choice paradigm was used. The reference interval contained the harmonic complex with a single modulated component at $1000 \mathrm{~Hz}$, and the target interval contained a similar harmonic complex in which the modulation rate was increased. Subjects were required to indicate the interval containing the higher modulation rate. A two-down one-up staircase procedure was used and threshold (corresponding to $71 \%$ correct) was defined as the mean difference in modulation rate at the last six reversals (Levitt, 1971). In this way a difference limen for modulation rate was defined for modulation rates of 2 and $16 \mathrm{~Hz}$, both of which fall within the range of beat rates used by tuners. A control task used a similar procedure to establish a difference limen for the frequency of an unmodulated $1000 \mathrm{~Hz}$ tone.

The task was designed to assess selective listening to envelope fluctuation of a single component within a harmonic complex as used by piano tuners. Such a skill is essential for piano tuning and was hypothesized to be enhanced in the tuners. The task does not simulate the process of navigating between notes that tuners must also carry out based on adjustment of the beats for a particular two-note intervals to a learned value. The AM discrimination data were analyzed to test the hypothesis that the piano tuners have a greater sensitivity to changes in envelope fluctuation at rates of 2 and $16 \mathrm{~Hz}$. We hypothesized that there would be no differences between the two groups for the tone frequency discrimination task.

All stimuli were created digitally using MATLAB (The MathWorks) at a sampling rate of $44.1 \mathrm{kHz}$ and 16 bit resolution and played to the subjects at a comfortable sound level.

Image acquisition. T1-weighted structural scans were acquired for the 19 piano tuners and 19 controls with a 1.5 Tesla Siemens Sonata MRI scanner using a three-dimensional MDEFT sequence (Deichmann et al., 2004 ) with the following acquisition parameters: time to repeat (TR), $20.66 \mathrm{~ms}$; time to echo (TE), $8.46 \mathrm{~ms}$; inversion time (TI), $640 \mathrm{~ms}$; flip angle, $\alpha, 25^{\circ}$; number of slices, 176 ; slice thickness, $1 \mathrm{~mm}$; isotropic voxels with dimensions, $1 \times 1 \times 1 \mathrm{~mm}^{3}$; matrix size, $256 \times 240($ read $\times$ phase $)$. The total duration of the scan was $\sim 9$ min.

Image processing. Imaging data were analyzed using Statistical Parametric Mapping (SPM8; Wellcome Trust Centre for Neuroimaging) and Diffeomorphic Anatomical Registration Through Exponentiated Lie algebra (DARTEL) (Ashburner, 2007), an image registration algorithm implemented in SPM to analyze structural data using voxel-based morphometry (VBM) (Ashburner and Friston, 2000). Each T1-weighted structural scan was spatially normalized into stereotactic space by coregistering with the standard MNI152 brain template. The coregistered images were segmented to obtain a bias-corrected structural image that has more uniform intensities within six different tissue classes, including gray matter (GM), white matter (WM), and CSF, and produces separate GM, WM, and CSF images. The GM and WM tissue images were imported into DARTEL to create Jacobian modulated tissue class images, and a series of template images were created by iteratively matching the images to align them with the average-shaped template. The final template obtained in this step was normalized to MNI space through an initial affine registration of the template with the tissue probability maps released with SPM8 (Friston et al., 1995b). The resultant images were smoothed with an isotropic Gaussian kernel of $8 \mathrm{~mm}$ at full-width at half-maximum and the GM volumes were computed using custom MATLAB scripts based on SPM8 functions. Region-of-interest (ROI) analysis was performed by defining a hippocampal ROI in the final average-shaped GM DARTEL template obtained from all subjects and 
A
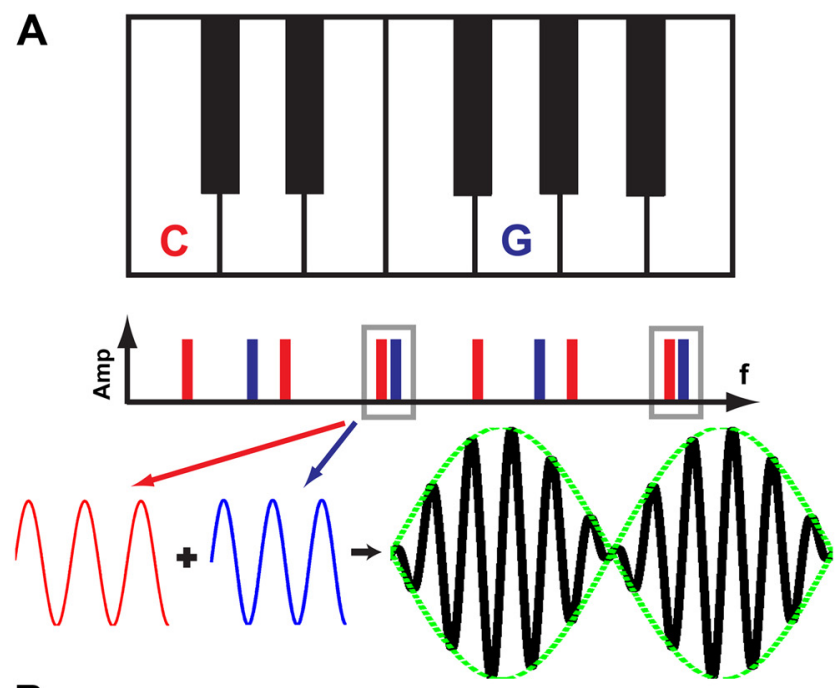

B

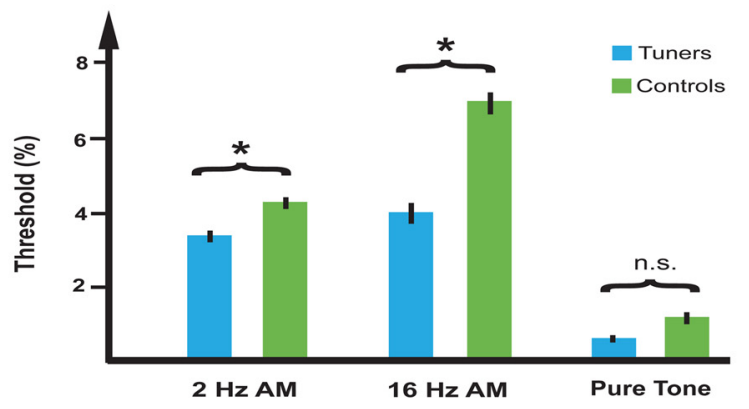

C
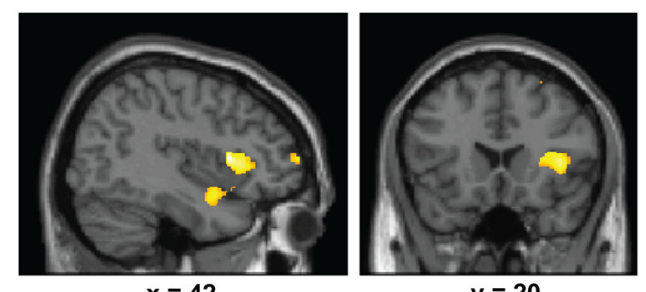

$y=20$

$\mathbf{t}$

Figure 1. Active listening to beats within particular spectral windows. $A$, Detection of beats in a spectral window for a two-note piano chord. A perfect fifth is shown between the notes $C$ (red) and $G$ (blue). This interval is so called because it spans five positions on the musical staff and corresponds to a seven-semitone pitch difference. When the popular equal-temperament tuning is used, in which the octave is divided into 12 exactly equal intervals, this corresponds to a pitch ratio of $2^{7 / 12}$ that is approximately, but not exactly, equal to 1.5. This means that there is near coincidence of the third (and sixth) harmonic of (and the second (and fourth) harmonic of G. Because the harmonics do not coincide exactly beating occurs between them. For clarity, the beat amplitudes are shown as having the same intensity: the actual spectrum of piano notes shows increasing attenuation of the higher harmonics. The time waveform of the third harmonic of $\mathrm{C}(\mathrm{red})$ and second harmonic of $\mathrm{G}$ (blue) are shown below where they interact to demonstrate beat production as indicated by the amplitude modulated black waveform (envelope shown in green). Piano tuners are therefore required to listen selectively to beat rates that only occur in particular spectral windows and to adjust the beat rates to prescribed values (typically $<20 \mathrm{~Hz}$ : Table 1) that specify the intervals. In this experiment, we tested a generic basis for this skill by requiring subjects to detect changing amplitude modulation of the third harmonic of a five-harmonic complex using beat rates within the range used by tuners (AM rate discrimination task). $B$, Mean thresholds for 19 piano tuners (depicted in blue) and 19 controls (depicted in green) are shown for the AM rate discrimination task at $2 \mathrm{~Hz}(3.26 \pm 0.32 \%$ vs $4.17 \pm 0.32 \%), 16 \mathrm{~Hz}(3.88 \pm$ $0.57 \%$ vs $6.93 \pm 0.60 \%)$, and a control task based on pure tone frequency discrimination $(0.44 \pm$ $0.10 \%$ vs $1.02 \pm 0.33 \%)$. Mean thresholds for the $2 \mathrm{~Hz}$ and $16 \mathrm{~Hz}$ AM tasks were significantly different between the two groups while there was no significant difference between groups on the frequency discrimination task (see Results). Error bars indicate \pm 1 SE. C, Enhanced GM volume in piano tuners was found in the right planum polare and frontal operculum as shown in the sagittal section at $x=42$. Greater GMvolume in the frontal operculum is shown in the coronal section at $y=20$. The strength of changes in GM volume ( $t$ value) is graded according to the color scheme on the right and shown at $p<0.001$ (uncorrected).

\section{A}

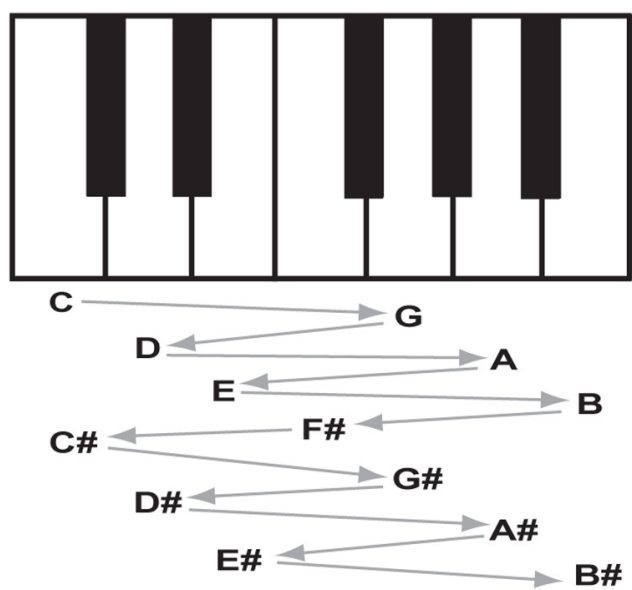

B
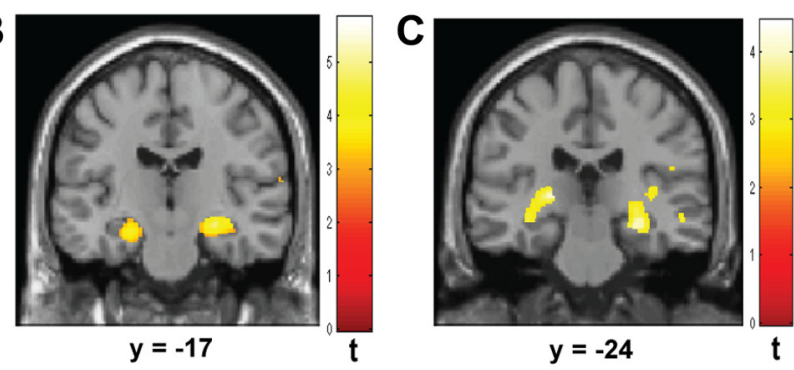

D

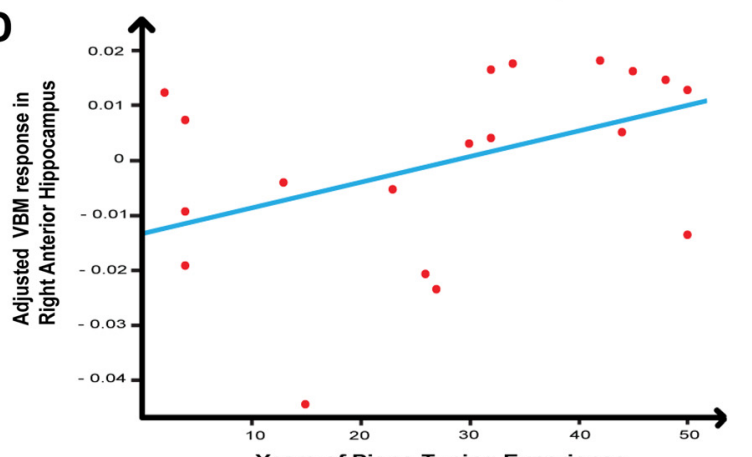

Figure 2. Navigation through a remembered piano soundscape. A, One algorithm for navigating the piano soundscape using successive two-note chords - a tuning sequence of rising perfect fifths and falling perfect fourths starting on C and ending on B\# immediately above the starting C. Different piano tuners use different schemes. $\boldsymbol{B}$, Significantly greater $G M$ volume as a function of years of piano tuning experience was found in the right anterior hippocampus as shown on a coronal section at $y=-17$. The significance of this change in $G M$ volume ( $t$ value) is graded according to the color scheme next to the figure and shown at $p<0.001$ (uncorrected). $C$, Significantly greater WM volume as a function of years of piano tuning experience was found in the posterior hippocampus bilaterally as shown on a coronal section at $y=-24$. The significance of this change in WM volume ( $t$ value) is graded according to the color scheme next to the figure and shown at $p<0.001$ (uncorrected). D, A significant positive correlation was found between the GM volume changes in the right anterior hippocampus and years of piano tuning $(r=0.43 ; p<0.05)$.

computing individual tuners' GM volumes within this ROI from their corresponding Jacobian modulated GM images.

Image analysis. Statistical analysis of the imaging data was conducted using the general linear model (Friston et al., 1995a) and appropriate $t$ tests using SPM8. The effects of age, gender, handedness, level of musical training, and total intracranial volume were removed by modeling them as confounding variables. We also examined the correlation of GM volume with years of piano tuning, musical training, and behavioral thresholds on the AM discrimination tasks for both piano tuners and controls. We checked whether there were any outliers as defined by three SDs from the mean; as there were none, all subjects were included in the analyses. 
VBM results are presented at a threshold of $p<0.001$ uncorrected for the regions we had an a priori hypothesis.

\section{Results}

We present here the results from the psychophysics and structural brain imaging study and consider the bases for the two essential stages of piano tuning: (1) active listening to beats within particular frequency windows (Fig. $1 A$ ) that may be a superior ability of the tuners in general (Fig. $1 B$ ) and results in specific changes in the structure of the tuners' brains (Fig. $1 C$ ) that is an "entry-level requirement" for the development of further tuning expertise, and, (2) the use of those beats to navigate through a remembered sound scene (Fig. $2 \mathrm{~A}$ ) that is a "second level" of expertise in the navigation of pitch space that can only develop after selective listening skills have been acquired.

\section{Active listening to beats}

We designed a psychophysical task based on amplitude modulation (AM) discrimination at modulation rates of 2 and $16 \mathrm{~Hz}$ to simulate detection of changes in beats in specific spectral windows and tested 19 piano tuners and an equal number of agematched control participants. This task simulates in a generic way a process that must be performed by tuners with any level of expertise to tune a piano: we hypothesized a categorical difference between the performance of tuners and controls and corresponding changes in the structure of the auditory cortex. ANOVA of threshold data with condition (2 Hz AM, $16 \mathrm{~Hz} \mathrm{AM}$, and pure-tone difference limens) as a within-subject measure and group (piano tuners vs controls) as a between-subject measure revealed a main effect of condition $\left(F_{(2,72)}=61.5, p<0.001\right)$ and group $\left(F_{(1,36)}=12.3, p<0.005\right)$ with a significant interaction between condition and group $\left(F_{(2,72)}=4.9, p<0.02\right)$.

The mean threshold for the $2 \mathrm{~Hz}$ AM discrimination task for the piano tuners was $3.26 \pm 0.32 \%$ while the mean threshold for the controls was $4.17 \pm 0.32 \%$ (Fig. $1 B$ ). A $t$ test revealed a significant difference $(p<0.05)$. The mean threshold for the 16 $\mathrm{Hz}$ AM discrimination task for the piano tuners was $3.88 \pm$ $0.57 \%$ while the mean threshold for the controls was $6.93 \pm$ $0.60 \%$. A $t$ test revealed a significant difference $(p<0.05)$. There was no significant difference between the pure-tone difference limen for the two groups.

There was no significant correlation between tuners' AM discrimination thresholds and years of tuning ( $2 \mathrm{~Hz}$ : $r=-0.018$, n.s. at $\alpha=0.05$; 16 Hz: $r=-0.343$, n.s. at $\alpha=0.05$ ) or starting age ( $2 \mathrm{~Hz}: r=0.083$, n.s. at $\alpha=0.05 ; 16 \mathrm{~Hz}: r=0.367$, n.s. at $\alpha=0.05)$.

To identify the anatomical bases for enhanced active listening skills, a categorical comparison of GM volume between piano tuners and controls was performed. Results demonstrate greater GM volume in tuners in right lateralized regions comprising the planum polare, frontal operculum (Fig. 1C), the superior frontal gyrus, and posterior cingulate gyrus (Table 2). Brain areas that showed reduced GM volume in the piano tuners as compared with controls include left hippocampus, parahippocampal gyrus, and superior temporal lobe (Table 3 ). There was no significant correlation between GM volume of these regions and tuning experience or between active listening task performance and years of tuning.

Overall, the present behavioral and structural results suggest a brain mechanism for active listening skills in the piano tuners that does not change appreciably with experience, and is demonstrated by a categorical comparison of behavior and structure between the piano tuners and controls. These results suggest that superior active listening skills are an early requirement for piano
Table 2. Brain areas with greater gray matter volume in piano tuners versus controls

\begin{tabular}{llrrrr}
\hline Brain area & Hemisphere & \multicolumn{1}{c}{$x$} & $y$ & \multicolumn{1}{c}{$z$} & $t$ value \\
\hline Frontal Operculum & Right & 42 & 14 & 7 & 4.85 \\
Planum polare & Right & 42 & 3 & -17 & 3.44 \\
Posterior Cingulate gyrus & Right & 6 & -25 & 34 & 3.59 \\
Superior frontal gyrus & Right & 11 & 59 & -6 & 3.68 \\
\hline
\end{tabular}

Local maxima are shown at $p<0.001$ (uncorrected).

Table 3. Brain areas with reduced grey matter volume in piano tuners versus controls

\begin{tabular}{llllll}
\hline Brain area & Hemisphere & $x$ & \multicolumn{1}{l}{$y$} & \multicolumn{1}{l}{$z$} & $t$ value \\
\hline Hippocampus & Left & -21 & -25 & -20 & 4.05 \\
Parahippocampal gyrus & Left & -36 & -12 & -20 & 3.70 \\
Superior temporal lobe & Left & -66 & -15 & 1 & 3.41 \\
\hline
\end{tabular}

Local maxima are shown at $p \leq 0.001$ (uncorrected).

Table 4. Brain areas showing increase in grey matter volume with years of piano tuning

\begin{tabular}{llrrrr}
\hline Brain area & Hemisphere & \multicolumn{1}{l}{$x$} & \multicolumn{1}{l}{$z$} & $t$ value \\
\hline Hippocampus & Right & 20 & -10 & -12 & 5.85 \\
& Left & -21 & -12 & -12 & 3.73 \\
Parahippocampal & Right & 27 & -21 & -14 & 5.33 \\
Gyrus & Left & -23 & -18 & -17 & 3.63 \\
Superior temporal gyrus & Right & 47 & -10 & -15 & 4.73 \\
Middle temporal gyrus & Right & 51 & -6 & -26 & 4.50 \\
Precuneus & Right & 8 & -42 & 57 & 4.65 \\
& Left & -3 & -43 & 63 & 4.49 \\
Insula & Right & 42 & 6 & -8 & 3.92 \\
& Left & -42 & 5 & -6 & 3.81 \\
Inferior & Right & 47 & -34 & 51 & 4.52 \\
Parietal lobe & Left & -36 & -51 & 57 & 3.81 \\
\hline
\end{tabular}

Local maxima are shown at $p<0.001$ (uncorrected).

Table 5. Brain areas showing increase in white matter volume with years of piano tuning

\begin{tabular}{lrrrr}
\hline Brain area & \multicolumn{1}{c}{$x$} & $y$ & \multicolumn{1}{l}{$z$} & $t$ value \\
\hline Right hippocampus & 30 & -24 & -12 & 3.98 \\
Left hippocampus & -33 & -27 & -8 & 4.05 \\
\hline
\end{tabular}

Local maxima are shown at $p \leq 0.001$ (uncorrected).

tuners that then allow the development of expert navigation through a complex soundscape.

\section{Navigation through the remembered auditory scene}

The behavioral characterization did not assess the second essential skill for piano tuning: navigation through a complex learned soundscape (Fig. 2A). This process requires previous memory formation, reorganization, and consolidation after learning in which the hippocampal complex has been implicated as an essential substrate (Squire, 2009). We hypothesized that there would be plastic changes in the hippocampus related to the continued accrual of acoustic navigational expertise over a lifetime of practice.

The analysis for correlation between GM volume and years of piano tuning experience revealed a specific increase of GM volume in the anterior hippocampus and parahippocampal gyrus bilaterally (Fig. $2 \mathrm{~B}$ ), as well as in the right middle temporal and superior temporal gyrus (STG), insula, precuneus, and the inferior parietal lobe (Table 4). The GM volume of these regions increased with years of tuning but not actual age. A similar anal- 
ysis for correlation between WM volume and years of tuning experience showed a specific increase of WM volume in the hippocampus bilaterally in a more posterior location to the increase in GM volume in the anterior hippocampus (Fig. 2C; Table 5).

A significant correlation was observed between GM volume (adjusted for differences in total intracranial volume) in the right anterior hippocampus and years of tuning $(r=0.43, p<0.05$; Fig. 2D). A stronger correlation with GM volume was obtained with years of tuning expressed as a percentage of actual age $(r=$ $0.48, p<0.05)$ and as a percentage of starting age $(r=0.60$, $p<0.005)$. The advantage due to early onset of training was confirmed by a strong negative correlation between GM volume and starting age $(r=-0.78, p<0.001)$.

These results were corroborated by an additional ROI-based analysis by defining a hippocampal ROI in the average-shaped GM template and computing individual tuners' GM volumes within this ROI. A significant correlation was obtained between GM volume in the right hippocampal ROI and years of tuning experience $(r=0.59, p<0.05)$.

The relationship between GM (and WM) volume and years of tuning took account of differences in musical expertise, and a separate analysis of the relationship between musical ability and GM volume suggested distinct areas in the temporal cortex that are congruent with previous studies. Musical training has been shown to lead to structural and functional changes in the inferior frontal and auditory cortex (Schneider et al., 2002; Bermudez and Zatorre, 2005; Hyde et al., 2009) and more recently in the hippocampus (Groussard et al., 2010; Herdener et al., 2010) but we found no correlation with musical training in the hippocampal areas showing a correlation between GM (and WM) volume and years of tuning.

\section{Discussion}

\section{Why study piano tuning?}

In the present study, we examined the underlying bases of acoustic expertise required for piano tuning. Tuning is a specialized profession and involves learning of theoretical concepts in acoustics as well as extensive practice (a taught degree course is available in the UK). Here, we tested professional tuners, who qualified as members of the Pianoforte Tuners' Association (UK) after a practical examination. Although these tuners had varying levels of musical expertise, musical skills such as absolute pitch are not considered essential or beneficial for expert tuning: tuning is based on the detection and adjustment of fundamental acoustic cues within a complex soundscape.

The unique nature of the tuning profession motivated us to study the acoustic bases of tuning processes and investigate neuroanatomical changes in the brain of the tuners that may develop over several years of repeated acoustic practice. Structural changes related to musical training have been studied previously, but the present subject group offers a rare opportunity to examine brain plasticity due to a specialized form of active listening that involves encoding of complex beat rate maps into memory and the use of these maps to navigate in pitch space.

\section{Learning to tune a piano}

Piano tuning requires two essential skills: (1) active listening to beats in particular frequency windows that depend on the interval being tuned, and (2) adjustment of the beat rates to certain specific values that depend both on the interval being tuned and the particular tuning algorithm or map used. Tuning is a sequential process that depends on the pitch "route" as the tuning of previously tuned notes affects the tuning of untuned notes. Thus, the beat rate to be adjusted varies according to the particular tuning path. The second process thus involves a complex form of mental navigation in pitch space using beat rates as landmarks. This process depends critically on whether the practitioner has learned and encoded beat rate values or "templates" for the different tuning algorithms. It is a complex procedure that involves use of pitch cues to navigate in conceptual space and retrieval of complex beat rate maps from memory.

Based on these two basic requirements of piano tuning, we predicted corresponding morphometric changes in brain areas involved in pitch analysis, and memory encoding and consolidation, respectively. These hypotheses emerge naturally from a bottom-up perspective based on a consideration of what piano tuners actually do. This leads naturally, in our view, to a two-stage mechanism for piano tuning where there are good scientific grounds to implicate the auditory cortex in the first stage of active listening and the hippocampus in the second stage, involving encoding and retrieval of beat rate maps associated with specific spectral contexts.

In the following subsections, we specifically discuss these two processes and the brain bases that support neural mechanisms relevant for active listening and navigation in remembered sound scenes.

\section{Active listening to beats}

In the absence of any previous scientific investigation of piano tuning, we carefully developed a psychophysical paradigm to simulate the acoustic processes involved in tuning in association with an expert piano tuner and instructor. Tuning is based on the detection of beats caused by simultaneous compression of two piano keys or an interval. This produces interaction between the constituent harmonics of the two notes that are close in frequency, leading to the modulation of the envelope at a rate given by the difference between the interacting harmonics. We simulated the process of listening to beats in a frequency window through the use of a five-element harmonic complex where only the middle component is modulated at a rate relevant to the tuning procedure (2 and $16 \mathrm{~Hz}$; Table 1 ).

The psychophysical results (Fig. $1 B$ ) demonstrate a superior and selective ability of piano tuners to carry out a task that mimics active listening for changes in beats in a frequency window. The active listening task requires perception, attention, and working memory. Functional imaging studies implicate mechanisms in the anterior and posterior superior temporal lobe in pitch analysis and working memory (Zatorre et al., 1994; Patterson et al., 2002; Overath et al., 2007). Right-lateralized temporal and frontal mechanisms for active listening are emphasized by functional imaging studies of normal participants (Zatorre et al., 1994) and anatomical studies of subjects with deficits in this ability (Hyde et al., 2007). Consistent with these active listening mechanisms, the piano tuners demonstrated greater GM volume in right lateralized frontotemporal regions, including the planum polare in the anterior temporal lobe and the frontal operculum (Table 2; Fig. 1C).

Although piano tuning is a specialized practice, precise analysis of sound envelopes is also critical for speech perception. The greater effect size for $16 \mathrm{~Hz}$ than $2 \mathrm{~Hz}$ (1.18 compared with 0.46 ) likely reflects the range of critical rates of envelope fluctuation for piano tuning (Table 1) extending to higher rates than those needed for speech analysis (Drullman et al., 1994; Chi et al., 1999). 


\section{Navigation in remembered sound scenes}

Tuners showed an experience-dependent increase in gray matter volume in a set of core regions including the anterior hippocampus, parahippocampal gyrus, parietal and superior temporal cortices, which may form part of an auditory memory network. Additionally, they also showed enhanced white matter volume in the posterior hippocampus as a function of years of tuning experience.

The present study is the first demonstration of hippocampal plasticity due to the prolonged execution of a fundamental acoustic task (the current group of 19 tuners had an average tuning experience of 27.7 years). The observed correlation with the GM volume in anterior hippocampus and parahippocampal gyrus suggests the importance of the medial temporal lobe (MTL) for auditory memory (Squire et al., 2001). These changes are likely related to the establishment of specific beat-rate templates, with different rates and spectral contexts for each interval tuned, which are retrieved during piano tuning. Repeated practice of piano tuning may lead to experience-dependent consolidation of these templates over time, which may facilitate fast recognition during online comparison and adjustment of beat rates.

Anatomical pathways underlying auditory memory from the superior temporal lobe to the hippocampal complex have been mapped extensively in primate models (Munoz-Lopez et al., 2010). The entorhinal cortex (EC) is the primary source of cortical projections to the hippocampus and receives direct and indirect projections via the adjacent perirhinal and parahippocampal cortices (Squire et al., 2004). Direct auditory projections to the EC originate in rostral and opercular parts of the STG (Amaral et al., 1983; Insausti and Amaral, 2008), insula, and inferior parietal cortex (Insausti et al., 1987, Insausti and Amaral, 2008), while the perirhinal and parahippocampal cortices also receive direct cortical projections from the STG and insula (Suzuki and Amaral, 1994; Blatt et al., 2003). MTL also receives indirect auditory input from the dorsal bank of the superior temporal sulcus, insula, and the inferior parietal cortex (Insausti et al., 1987; Suzuki and Amaral, 1994). The present correlation between years of experience and GM volume in the parietal cortex is congruent with its functional role in the mental rotation of melodies (Zatorre et al., 2010) as an alternative type of manipulation in pitch space. The current data and these anatomical observations provide a structural basis for expert piano tuning that includes a set of linked areas in the parietal, superior lateral temporal lobe, and the anterior hippocampus in the MTL.

The hippocampus contains mechanisms for spatial navigation (O'Keefe and Dostrovsky, 1971) that are theoretically emphasized (O'Keefe and Nadel, 1978; Moser et al., 2008), and this role has been corroborated using structural brain imaging to demonstrate a similar degree of correlation between experience in spatial navigation (taxi driving) and GM volume in distinct regions of the hippocampus in a more posterior location (Maguire et al., 2000; Woollett and Maguire, 2011). In this study we have studied bases for a skill that has some formal similarities to spatial navigation, where experts are required to perceive and relate local acoustic features to a complex soundscape containing many other features and move between different acoustic scenes according to the local features detected (beats). The present data therefore suggest a role for the hippocampus in navigation and relational organization of remembered events in conceptual space (MacDonald et al., 2011), which could be based on similar neural architecture to that used for navigation in physical space.

\section{Conclusions}

We studied a previously untested group of professional piano tuners who possess rare skills in detecting beat rates at particular frequencies across a wide range of piano notes. Within the group of piano tuners, we found that GM and WM volume increased with tuning experience in the anterior hippocampus and superior lateral temporal lobe and the posterior hippocampus, respectively. The results suggest a role for this hippocampo-cortical network in skilled exploration of complex soundscapes in which intricate beat rate templates are encoded and consolidated into memory over time in an experience-dependent manner.

\section{Notes}

Supplemental material for this article is available at http://www.fil.ion. ucl.ac.uk/ steki/Piano_Tuners.zip. Audio examples of $2 \mathrm{~Hz}$ AM and 16 $\mathrm{Hz}$ AM stimuli used in psychophysics. This material has not been peer reviewed.

\section{References}

Amaral DG, Insausti R, Cowan WM (1983) Evidence for a direct projection from the superior temporal gyrus to the entorhinal cortex in the monkey. Brain Res 275:263-277.

Ashburner J (2007) A fast diffeomorphic image registration algorithm. Neuroimage 38:95-113.

Ashburner J, Friston KJ (2000) Voxel-based morphometry-the methods. Neuroimage 11:805-821.

Bermudez P, Zatorre RJ (2005) Differences in gray matter between musicians and nonmusicians. Ann NY Acad Sci 1060:395-399.

Blatt GJ, Pandya DN, Rosene DL (2003) Parcellation of cortical afferents to three distinct sectors in the parahippocampal gyrus of the rhesus monkey: an anatomical and neurophysiological study. J Comp Neurol 466:161-179.

Capleton B (2007) Theory and practice of piano tuning. Malvern, UK: Amarilli Books.

Chi T, Gao Y, Guyton MC, Ru P, Shamma S (1999) Spectro-temporal modulation transfer functions and speech intelligibility. J Acoust Soc Am 106:2719-2732.

Deichmann R, Schwarzbauer C, Turner R (2004) Optimisation of the 3D MDEFT sequence for anatomical brain imaging: technical implications at 1.5 and 3 T. Neuroimage 21:757-767.

Drullman R, Festen JM, Plomp R (1994) Effect of temporal envelope smearing on speech reception. J Acoust Soc Am 95:1053-1064.

Friston KJ, Holmes AP, Worsley KJ, Poline J, Frith CD, Frackowiak RS (1995a) Statistical parametric maps in functional imaging: a general linear approach. Hum Brain Mapp 2:189-210.

Friston KJ, Ashburner J, Frith CD, Poline J, Heather JD, Frackowiak RSJ (1995b) Spatial registration and normalization of images. Hum Brain Mapp 3:165-189.

Groussard M, La Joie R, Rauchs G, Landeau B, Chételat G, Viader F, Desgranges B, Eustache F, Platel H (2010) When music and long-term memory interact: effects of musical expertise on functional and structural plasticity in the hippocampus. PLoS ONE 5:e13225.

Herdener M, Esposito F, di Salle F, Boller C, Hilti CC, Habermeyer B, Scheffler K, Wetzel S, Seifritz E, Cattapan-Ludewig K (2010) Musical training induces functional plasticity in human hippocampus. J Neurosci 30:1377-1384.

Hyde KL, Lerch JP, Zatorre RJ, Griffiths TD, Evans AC, Peretz I (2007) Cortical thickness in congenital amusia: when less is better than more. J Neurosci 27:13028-13032.

Hyde KL, Lerch J, Norton A, Forgeard M, Winner E, Evans AC, Schlaug G (2009) Musical training shapes structural brain development. J Neurosci 29:3019-3025.

Insausti R, Amaral DG (2008) Entorhinal cortex of the monkey: IV. Topographical and laminar organization of cortical afferents. J Comp Neurol 509:608-641

Insausti R, Amaral DG, Cowan WM (1987) The entorhinal cortex of the monkey: II. Cortical afferents. J Comp Neurol 264:356-395.

Levitt H (1971) Transformed up-down methods in psychoacoustics. J Acoust Soc Am 49 [Suppl 2]:467-477.

MacDonald CJ, Lepage KQ, Eden UT, Eichenbaum H (2011) Hippocampal 
"time cells" bridge the gap in memory for discontiguous events. Neuron 71:737-749.

Maguire EA, Gadian DG, Johnsrude IS, Good CD, Ashburner J, Frackowiak RS, Frith CD (2000) Navigation-related structural change in the hippocampi of taxi drivers. Proc Natl Acad Sci U S A 97:4398-4403.

Moser EI, Kropff E, Moser MB (2008) Place cells, grid cells, and the brain's spatial representation system. Annu Rev Neurosci 31:69-89.

Munoz-Lopez MM, Mohedano-Moriano A, Insausti R (2010) Anatomical pathways for auditory memory in primates. Front Neuroanat 4:129.

O'Keefe J, Dostrovsky J (1971) The hippocampus as a spatial map. Preliminary evidence from unit activity in the freely-moving rat. Brain Res 34:171-175.

O'Keefe J, Nadel L (1978) The hippocampus as a cognitive map. Oxford: Oxford UP.

Overath T, Cusack R, Kumar S, von Kriegstein K, Warren JD, Grube M, Carlyon RP, Griffiths TD (2007) An information theoretic characterisation of auditory encoding. PLoS Biol 5:e288.

Patterson RD, Uppenkamp S, Johnsrude IS, Griffiths TD (2002) The processing of temporal pitch and melody information in auditory cortex. Neuron 36:767-776.
Schneider P, Scherg M, Dosch HG, Specht HJ, Gutschalk A, Rupp A (2002) Morphology of Heschl's gyrus reflects enhanced activation in the auditory cortex of musicians. Nat Neurosci 5:688-694.

Squire LR (2009) Memory and brain systems: 1969-2009. J Neurosci 29:12711-12716.

Squire LR, Schmolck H, Stark SM (2001) Impaired auditory recognition memory in amnesic patients with medial temporal lobe lesions. Learn Mem 8:252-256.

Squire LR, Stark CE, Clark RE (2004) The medial temporal lobe. Annu Rev Neurosci 27:279-306.

Suzuki WA, Amaral DG (1994) Perirhinal and parahippocampal cortices of the macaque monkey: Cortical afferents. J Comp Neurol 350:497-533.

Woollett K, Maguire EA (2011) Acquiring "the knowledge" of London's layout drives structural brain changes. Curr Biol 21:2109-2114.

Zatorre RJ, Evans AC, Meyer E (1994) Neural mechanisms underlying melodic perception and memory for pitch. J Neurosci 14:1908-1919.

Zatorre RJ, Halpern AR, Bouffard M (2010) Mental reversal of imagined melodies: a role for the posterior parietal cortex. J Cogn Neurosci 22:775789. 